\title{
Frictional Drag between Two Dilute Two-Dimensional Hole Layers
}

\author{
R. Pillarisetty, Hwayong Noh, D. C. Tsui, E. P. De Poortere, E. Tutuc, and M. Shayegan \\ Department of Electrical Engineering, Princeton University, Princeton, NJ 08544
}

(October 28, 2018)

\begin{abstract}
We report drag measurements on dilute double layer two-dimensional hole systems in the regime of $r_{s}=19 \sim 39$. We observed a strong enhancement of the drag over the simple Boltzmann calculations of Coulomb interaction, and deviations from the $T^{2}$ dependence which cannot be explained by phonon-mediated, plasmon-enhanced, or disorder-related processes. We suggest that this deviation results from interaction effects in the dilute regime.
\end{abstract}

73.40.-c,73.21.Ac, $73.40 . \mathrm{Kp}$

In recent years, transport properties in dilute twodimensional (2D) electron or hole systems have generated much interest from the possibility that strong interactions between charge carriers in such systems could stabilize a new quantum state, which can exhibit unusual transport characteristics. In contradiction to the scaling theory of localization [1], which predicts only an insulating state in $2 \mathrm{D}$ in the absence of interactions, a metallic behavior characterized by a decreasing resistivity $(\rho)$ with decreasing temperature $(T)$ and an apparent metalinsulator transition as the carrier density is varied has been observed in many dilute 2D systems [2]. Despite numerous experimental and theoretical studies, there is no conclusive understanding of the nature of the metallic behavior at this point. It is an interesting question whether this metallic behavior is related to any non-Fermi liquid behavior, since the interactions between the carriers are strong. Studies [3] indicate that the 2D system behaves as a Fermi liquid even in this dilute metal-insulator transition regime by examining the weak localization and interaction corrections to the conductivity. In this paper, we present an alternative way of studying the validity of Fermi liquid theory in this dilute regime: frictional drag experiment.

Drag measurements, which are performed on double layer structures, have been utilized to study the interlayer carrier-carrier interactions [4,5]. These measurements are done by passing a drive current $\left(I_{\text {drive }}\right)$ through one of the layers, while measuring the potential difference $\left(V_{D}\right)$, which arises in the other layer due to carrier momentum transfer. The drag resistivity, given by $\rho_{D}=(W / L) V_{D} / I_{\text {drive }}$, where $W$ and $L$ are the width and the length of the sample, provides a direct measure of the interlayer scattering rate in these structures. The first component of $\rho_{D}$ has been identified as from the Coulomb interaction, which is characterized by a quadratic increase of $\rho_{D}$ with $T$ in a simple single particle picture [4].6.6]. Any deviations from this $T^{2}$ dependence would mean either a non-Fermi liquid behavior or an additional interaction mechanism. Most previous drag measurements have been performed on high density samples, and confirmed the $T^{2}$ dependence when the layer separation was $300 \AA$ and less [7], while they showed deviations from this dependence [4, $8,10,12]$ when either the layer separation is large or the temperature is high. These deviations, shown as a peak when $\rho_{D} / T^{2}$ is plotted as a function of $T$, were attributed to either a phonon-mediated process [4 4 11] or a plasmon excitation contribution 12, 13.

What is of major interest in our study is the drag in the low density regime with small layer separation in order to investigate the validity of $T^{2}$ dependence in the $T \rightarrow 0$ limit, a test for the Fermi liquid behavior. In this paper, we present drag measurements on dilute two dimensional hole systems at layer densities $(p)$ ranging from $2.5 \times 10^{10} \mathrm{~cm}^{-2}$ to $6 \times 10^{9} \mathrm{~cm}^{-2}$. These are the first reported results, to our best knowledge, of drag measurements in this low density range. In addition, the larger effective mass $\left(m^{*}\right)$ in the hole samples allows us to explore a much higher $r_{s}$ regime than has been previously measured in electron samples; $r_{s}$ is the ratio of interaction energy to Fermi energy and is given by $r_{s}=(p \pi)^{-1 / 2} m^{*} e^{2} / 4 \pi \hbar^{2} \epsilon \epsilon_{0}$, where $\epsilon$ is the dielectric constant. The $r_{s}$ values of the $2 \mathrm{D}$ systems which exhibit metal-insulator transition is usually around 10 or higher at the transition. Using the hole effective mass for our system $m^{*}=0.38 m_{e}$, we find that the $r_{s}$ values for our measurements range from 19 to 39. This is more than an order of magnitude larger than the $r_{s}$ values for the lowest density electron systems which have been measured [7]. From the $T$ dependence of $\rho$ taken from one of the layers, we observed a clear metal-insulator transition at $p \sim 8.5 \times 10^{9} \mathrm{~cm}^{-2}$ and $\rho \sim 30 k \Omega / \square$ (inset of Fig. 11).

Our drag measurements in this regime show that, for $T \leq 0.5 T_{F}$, the drag exhibits a slightly stronger than $T^{2}$ dependence, where $T_{F}$ is the Fermi temperature. The magnitude of the drag is much larger than the simple Boltzmann calculations predict [6]. As the temperature is further increased we find a crossover to a sublinear dependence, and when $\rho_{D} / T^{2}$ is plotted as a function of $T$, the data exhibits a peak similar to that in a phonon-mediated [48 11] or plasmon-enhancement process [12,13]. However, from the dependence on the density ratio of the two layers, we observe no evidence of such processes in our systems. In addition, we find our data to be inconsistent with previous disorder enhanced studies 
of the drag 14. These findings lead us to believe that interaction effects are behind the observed enhancement.

The samples used in this experiment are Si $\delta$-doped AlGaAs/GaAs/AlAs double quantum well structures, which were grown by molecular beam epitaxy on the (311)A surface. Most of this paper presents data taken from a low density double layer hole sample, which we will refer to as sample A. We also provide data from an intermediate density double layer hole sample, which we refer to as sample B. The structure of sample A (sample B) consists of two 150 (175) $\AA$ GaAs quantum wells separated by a 150 (100) A AlAs barrier, corresponding to a center to center layer separation $(d)$ of $300(275) \AA$. The grown densities in each layer are $2.5 \times 10^{10} \mathrm{~cm}^{-2}$ for sample A, and $7.0 \times 10^{10} \mathrm{~cm}^{-2}$ for sample B. For sample $\mathrm{A}$, the mobility of each layer is roughly $1.5 \times 10^{5} \mathrm{~cm}^{2} / \mathrm{Vs}$ for the grown densities at $300 \mathrm{mK}$. For sample B, the mobilities are, for the grown densities, $5.7 \times 10^{5} \mathrm{~cm}^{2} / \mathrm{Vs}$ and $7.7 \times 10^{5} \mathrm{~cm}^{2} / \mathrm{Vs}$ for the top and bottom layer respectively, at $300 \mathrm{mK}$. The samples were processed using a selective depletion scheme allowing independent contact to each of the two layers [15]. Both layer densities are independently tunable with the aid of gates evaporated on both sides of the sample.

The data presented in this paper were taken in $a{ }^{3} \mathrm{He}$ refrigerator and in a dilution refrigerator. The layer densities were determined by independently measuring Shubnikov-de Haas oscillations of each layer. Drive currents between $500 \mathrm{pA}$ to $10 \mathrm{nA}$ were passed through one of the layers, while the drag signal was measured in the other layer, using standard lock-in techniques at 1 to 4 $\mathrm{Hz}$. We performed all the standard consistency checks on our measurements [4. The grounding to the drag layer was swapped between voltage contacts and the signals matched. The drag and drive layers were swapped and showed good agreement. The drag signal showed a linear variation with the drive current. Also, the out of phase signal was monitored to ensure that capacitive coupling between the layers was not affecting our measurement. It should be pointed out that three working samples were made from wafer A and two from wafer B. All of the samples from each wafer showed reasonable agreement with each other. However, data are presented from only one sample from each wafer.

In Fig. 1, we present $\rho_{D}$ vs $T$ for sample A at $p=$ $2.5 \times 10^{10} \mathrm{~cm}^{-2}$. The temperatures in this plot range from $0.08 T_{F}$ to $0.25 T_{F}$. When a trial fit is made with $T^{2}$ (dashed line), the data does not show good agreement. Instead, when we fit the data with $T^{2.5}$ (solid line), a better result is obtained. When the exponent is varied as a free parameter, we also find a value close to 2.5. It is clear that at low temperatures, $\rho_{D}$ shows a stronger than $T^{2}$ dependence. In addition, we find a very large enhancement of $\rho_{D}$. For this density, our measurements are greater than 200 times that calculated from the Boltzmann equation [6] (valid for $k_{F} d \gg 1$ and

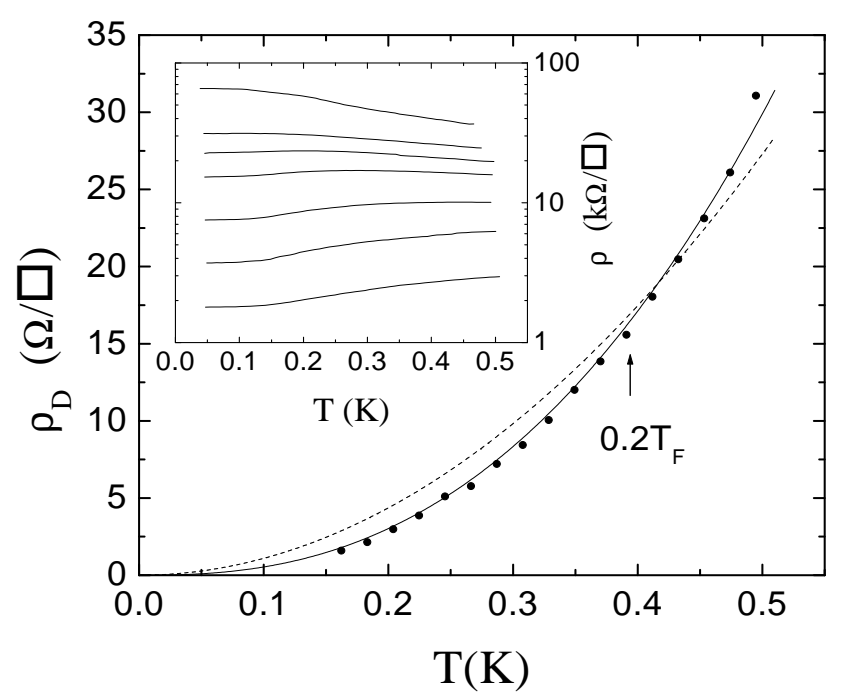

FIG. 1. $\rho_{D}$ vs $T$ for sample A. Layer densities are matched at $p=2.5 \times 10^{10} \mathrm{~cm}^{-2}$. Provided are the best $T^{2}$ (dashed line) and $T^{2.5}$ (solid line) fits. Inset: $\rho$ vs $T$ for the bottom layer. From top to bottom, $p=0.80,0.85,0.90,1.0,1.2,1.4$, and $2.0 \times 10^{10} \mathrm{~cm}^{-2}$.

$T \ll T_{F}$, with $k_{F} d=1.2$ and $T<0.25 T_{F}$ in our measurement) and roughly 5 orders of magnitude larger than the original drag experiment involving high density electrons $\left(n=1.5 \times 10^{11} \mathrm{~cm}^{-2}\right)$ with $d=375 \AA\left(\rho_{D}=0.5 \mathrm{~m} \Omega / \square\right.$ at $500 \mathrm{mK}$ ) [4. More recent measurements on bilayer electron systems [7], which have similar $d$ to our samples, showed a nearly quadratic temperature dependence for densities ranging from $5.3 \times 10^{10} \mathrm{~cm}^{-2}$ to $2.5 \times 10^{10}$ $\mathrm{cm}^{-2}$, which are slightly larger than ours samples. For the largest density, they measured $\rho_{D} / T^{2} \sim 0.4 \Omega / \square K^{2}$, which is only four times larger than that expected from a Boltzmann calculation $\left(k_{F} d=1.6\right.$, which is comparable to our sample). It is evident that Boltzmann transport theory fails to provide either the proper temperature dependence or the correct magnitude for measurement in our samples.

In Fig. 2, we present the complete temperature dependence of $\rho_{D}$ for matched layer densities at $p=1.5 \times 10^{10}$ $\mathrm{cm}^{-2}$ taken from sample A. Although the data points on the low temperature side are again well described by a $T^{2.5}$ fit (dashed line), as the temperature is further increased we find a crossover to a sub-linear dependence at approximately $0.5 T_{F}$. Also, we find the magnitude at this density to be roughly 500 times larger than the Boltzmann calculation [6]. For comparison we also present intermediate density data taken from sample B. For this sample $d=275 \AA$, so comparison is reasonable. For matched layer densities, $p=7 \times 10^{10} \mathrm{~cm}^{-2}$, the data exhibits a nearly quadratic temperature dependence, and has a magnitude about 20 times larger than that calculated from the Boltzmann equation [6]. At this intermediate density, we find no evidence that the system cannot 
be described by Fermi liquid theory. Note that this data shows no significant deviation from $T^{2}$ up to and above the Fermi temperature of about $5 \mathrm{~K}$. This is in contrast to the low density sample (sample A), which clearly exhibits non-quadratic behavior at temperatures far below $T_{F}$.

The nature of this deviation from the $T^{2}$ dependence in the dilute regime is of much importance, since it possibly could be representative of non-Fermi liquid behavior of the strongly interacting charge carriers. On the other hand, it is also possible that the system can still be described as a Fermi liquid and the deviations from $T^{2}$ arise from the activation of some other interlayer momentum transfer mechanism. We approach this question by examining the deviation from the simple quadratic dependence more closely. In Fig. 3, we present the $T$ dependence of $\rho_{D} / T^{2}$. Data is shown for layer densities from $2.5 \times 10^{10} \mathrm{~cm}^{-2}$ to below $1 \times 10^{10} \mathrm{~cm}^{-2}$. The deviation is clearly demonstrated by a local maxima around a characteristic crossover temperature, similar to previous drag measurements involving phonon mediated [4 8 [10] and plasmon enhanced processes 12].

For electron scattering mediated by phonons, this deviation arises from scattering processes involving $2 k_{F}$ phonons, and the expected peak in $\rho_{D} / T^{2}$ vs $T$ was observed in higher density samples near the BlochGrüneisen temperature $\left(T_{B G}\right)$, given by $k_{B} T_{B G}=$ $2 \hbar k_{F} c_{s}$, where $c_{s}$ is the sound velocity in the barrier [8 10]. For our sample, $T_{B G}$ ranges from 1 to $2 \mathrm{~K}$, and

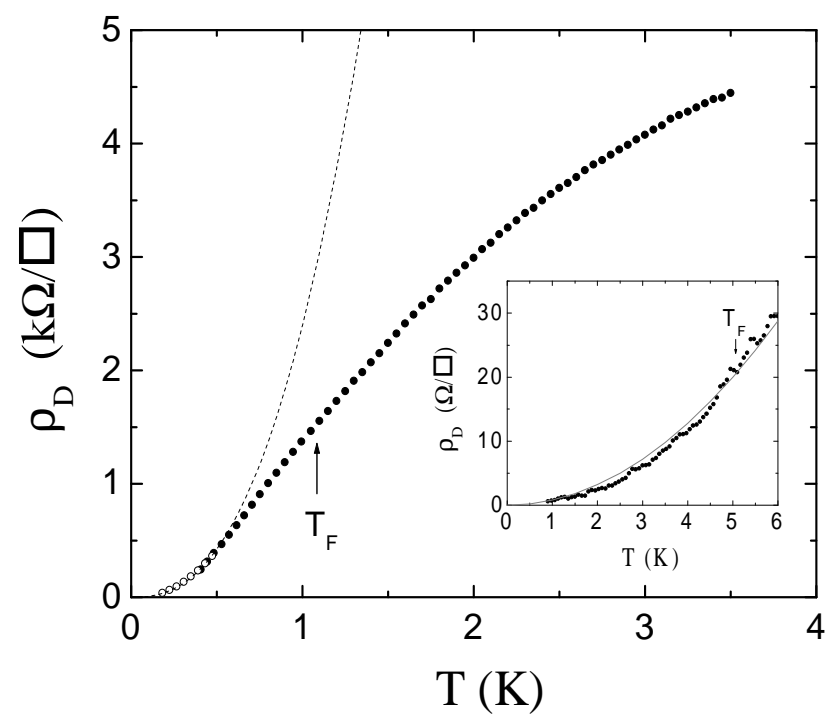

FIG. 2. $\rho_{D}$ vs $T$ for sample A. Layer densities are matched at $p=1.5 \times 10^{10} \mathrm{~cm}^{-2}$. Data measured in the dilution refrigerator are shown with open circles. Also shown is the best $T^{2.5}$ fit of these points. Inset: $\rho_{D}$ vs $T$ for sample B, whose layer densities are matched at $p=7.0 \times 10^{10} \mathrm{~cm}^{-2}$. Also shown is the best $T^{2}$ fit of the data. The Fermi temperature is indicated on both plots. the phonon-mediated process is certainly a possibility. In previous experiments on electron samples, a second peak in $\rho_{D} / T^{2}$ was observed at still higher temperatures near $0.5 T_{F}$, which arises from a plasmon excitation 12]. Since $0.5 T_{F}$ in our low density sample ranges from $500 \mathrm{mK}$ to $1.5 \mathrm{~K}$, we expect the peak positions arising from both phonon and plasmon processes to fall in roughly the same range. Therefore, we look at the density dependence of the peak position to determine which of these could be contributing to $\rho_{D}$.

In the inset (a) of Fig. 3, we plot the peak position versus the matched layer density. It is clear that the peak position shifts to lower temperature as the density is lowered. We expect, for phonon mediated processes, this peak should scale with the square root of the matched density. On the other hand for plasmon enhanced processes, the peak should scale with the matched layer density. To determine whether the peak in the scaled drag

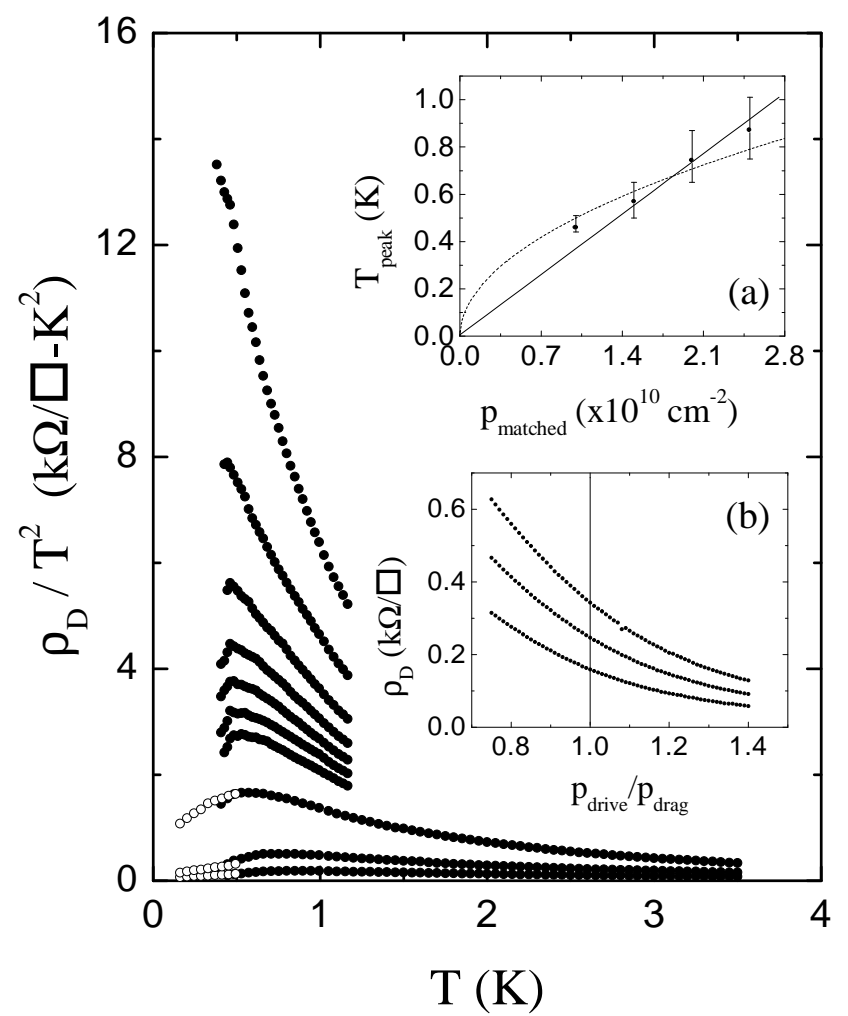

FIG. 3. $\rho_{D} / T^{2}$ vs $T$ for sample A. Data measured in the dilution refrigerator are shown as open circles. From top to bottom, the layer densities $\left(p_{\text {drive }} / p_{\text {drag }}\right)$ in $10^{10} \mathrm{~cm}^{-2}$ are $0.6 / 1.0,0.8 / 1.0,1.0 / 1.0,1.0 / 1.3,1.1 / 1.4,1.2 / 1.4,1.5 / 1.5$, 2.0/2.0, 2.5/2.5. Inset: (a) Peak position temperature vs matched layer density. Also provided are the best linear (solid line) and square root (dashed line) fits of the data. (b) $\rho_{D}$ vs density ratio at (from top to bottom) $T=860,730$, and $600 \mathrm{mK}$. The drag layer is fixed at $2.0 \times 10^{10} \mathrm{~cm}^{-2}$, while the drive layer is swept from 2.4 to $1.5 \times 10^{10} \mathrm{~cm}^{-2}$, passing through the matched density point indicated by the solid line. 
resistivity could be arising from phonon or plasmon processes, we provide the best square root fit (phonon) and the best linear fit (plasmon) of the data. Considering the error bar associated with the determination of peak position and the narrow range of data, we found that the precise density dependence of this peak position cannot be specified accurately.

Yet, regardless of whether phonon or plasmon processes contribute to the drag resistivity, this enhancement should be strongest when the layer densities are matched. In the former, phonon exchange between carriers in each layer mediates the scattering process, and one would expect the enhancement of the drag resistivity to be largest when the $2 k_{F}$ phonons in each layer are identical. Drag measurements on the high density electron samples exhibited a sharp local maxima when the densities were matched [8,9]. Similar to the phonon case, the plasmon enhancement of the drag resistivity was also shown to be maximized when the layer densities were matched [12]. In the inset (b) of Fig. 3, we present measurements of $\rho_{D}$ versus density ratio of the two layers. The drive layer density was slowly changed for a fixed drag layer density of $2.0 \times 10^{10} \mathrm{~cm}^{-2}$, and the resulting drag signal was measured. The measurements were performed near the peak temperature for matched density of $2.0 \times 10^{10}$ $\mathrm{cm}^{-2}$. We notice that the density dependence is monotonic and we see no structure at all anywhere around the matched density point. Although we may not expect a strong peak at the matched density because the Fermi temperature difference between the two layers are about the same as the measurement temperature in this plot, we note that the previous plasmon-enhanced drag measurements [12] showed a peak under similar conditions. If the thermal broadening makes the peak invisible for the phonon-mediated process, we expect that the peak in the temperature dependence in Fig. 3 would be broadened significantly as well, which is not the case in our data.

From the density dependence data, we find our measurements are inconsistent with the results of previous drag experiments involving phonons and plasmons. In fact, no evidence of either of these were seen in our intermediate density sample (Fig. 2 inset), and there is no reason to believe that these mechanisms should become much stronger as the density is lowered. On the other hand, we know that as the density is tuned into the dilute regime both disorder and interaction effects play an important role in charge transport. In the diffusive limit as $T \rightarrow 0$, the disorder is expected to enhance the drag, and change the temperature dependence from the expected $T^{2}$ to $-T^{2} \ln T$ [14]. Investigating the possibility that the deviation from the $T^{2}$ dependence in our low temperature data could be due to diffusive behavior, we found that the data could not be fit by $-T^{2} \ln T$. In addition, the inset (a) of Fig. 3 shows that the boundary of the regime of the enhancement to the $T^{2}$ dependence shifts to lower temperature as the density and, in turn, the mobility is lowered. This is in contrast to the expectation that the boundary of the diffusive behavior should scale with the disorder temperature of the system, given by $\hbar / \tau$, where $\tau$ is the transport scattering time, and it should shift to higher temperatures as the mobility is lowered. These inconsistencies lead us to believe that no signatures of the disorder are evident in our data.

In conclusion, we find ourselves unable to explain the drag measurements in this dilute regime by accounting for additional corrections due to phonon, plasmon or disorder enhanced processes. We are therefore led to believe that the characteristics observed in the data are due to interaction effects. Calculations 16 have shown that for electron systems with intermediate densities, $\rho_{D}$ still exhibits a $T^{2}$ dependence at low temperatures and is enhanced by an order of magnitude over the Boltzmann calculations by accounting for intralayer correlations. However, we find that this calculation can only partly explain our data since we observe a much larger enhancement and a greater than $T^{2}$ dependence. We therefore find it still unclear whether the strong interaction between carriers simply adds more corrections which enhance and alter the temperature dependence of the drag, or if the interaction stabilizes a new state which exhibits non-Fermi liquid behavior.

We acknowledge S. Das Sarma, S. Simon, and T. J. Gramila for useful discussions related to the data. This work is supported by the NSF, and a DURINT grant through the ONR.

[1] E. Abrahams, P.W. Anderson, D.C. Licciardello, and T.V. Ramakrishnan, Phys. Rev. Lett. 42, 673 (1979).

[2] For a recent review see B.L. Altshuler, D.L. Maslov, and V.M. Pudalov, Physica E (Amsterdam) 9(2), 209 (2001); E. Abrahams, S.V. Kravchenko, and M.P. Sarachik, Rev. Mod. Phys. 73, 251 (2001).

[3] M.Y. Simmons et al., Phys. Rev. Lett. 84, 2489 (2000).

[4] T.J. Gramila et al., Phys. Rev. Lett. 66, 1216 (1991).

[5] See for a review A.G. Rojo, J. Phys.: Condens. Matter 11, R31 (1999).

[6] A. Jauho and H. Smith, Phys. Rev. B 47, 4420 (1993).

[7] M. Kellogg et al., cond-mat/0108403.

[8] T.J. Gramila et al., Phys. Rev. B 47, 12957 (1993).

[9] H. Rubel et al., Semicond. Sci. Technol. 10, 1229 (1995); H. Noh et al., Phys. Rev. B 59, 13114 (1999).

[10] C. Jörger et al., Physica E 6, 598 (2000).

[11] M. Bønsager et al., Phys. Rev. B 57, 7085 (1998).

[12] N.P.R. Hill et al., Phys. Rev. Lett. 78, 2204 (1997); H. Noh et al., Phys. Rev. B 58, 12621 (1998).

[13] K. Flensberg and B.Y.-K. Hu, Phys. Rev. Lett. 73, 3572 (1994).

[14] L. Zheng and A.H. MacDonald, Phys. Rev. B 48, 8203 (1993).

[15] J.P. Eisenstein, L.N. Pfieiffer, and K.W. West, Appl. Phys. Lett. 57, 2324 (1990).

[16] L. Świerkowski, J. Szymański, and Z.W. Gortel, Phys. Rev. B 55, 2280 (1997). 